\title{
Ambipolar quantum transport in few-layer black phosphorus
}

\author{
Gen Long, ${ }^{1}$ Denis Maryenko, ${ }^{2}$ Sergio Pezzini, ${ }^{3}$ Shuigang Xu, ${ }^{1,4}$ Zefei Wu, ${ }^{1}$ Tianyi Han,,${ }^{1}$ Jiangxiazi Lin,,${ }^{1}$ Chun Cheng, ${ }^{5}$ \\ Yuan Cai, ${ }^{1}$ Uli Zeitler, ${ }^{3}$ and Ning Wang ${ }^{1, *}$ \\ ${ }^{1}$ Department of Physics and Center for Quantum Materials, The Hong Kong University of Science and Technology, Hong Kong, China \\ ${ }^{2}$ RIKEN Center for Emergent Matter Science (CEMS), Wako 351-0198, Japan \\ ${ }^{3}$ High Field Magnet Laboratory (HFML-EMFL) and Institute for Molecules and Materials, Radboud University, \\ 6525 ED Nijmegen, The Netherlands \\ ${ }^{4}$ National Graphene Institute, University of Manchester, Manchester M13 9PL, United Kingdom \\ ${ }^{5}$ Department of Materials Science and Engineering, and Shenzhen Key Laboratory of Nanoimprint Technology, \\ South University of Science and Technology, Shenzhen 518055, China
}

(Received 22 May 2017; revised manuscript received 9 October 2017; published 27 October 2017)

\begin{abstract}
We have investigated the quantum transport properties of high-mobility electrons and holes in atomically thin black phosphorus ambipolar devices. The two-dimensional hole system exhibits unambiguously the quantum Hall effect in a magnetic field up to $30 \mathrm{~T}$, while the electron system shows clearly developing Hall plateaus at integer Landau level filling factors accompanied by $R_{x x}$ oscillations, signaling the onset of the quantum Hall effect. By bringing the spin-resolved Landau levels of the electron system to a coincidence, we determine an electron spin susceptibility to be $\chi_{s e}=m^{*} g^{*}=1.1 \pm 0.03$, which, combined with the electron mass $m^{*}=$ $0.39 m_{0}$, yields a Landé $g$ factor $g^{*}=2.8 \pm 0.2$. The enhancement of spin susceptibility in the black phosphorus two-dimensional electron system is around $50 \%$ compared with band susceptibility, which agrees well with various two-dimensional charge-carrier systems with weak spin-orbit coupling, suggesting the important role played by the exchange interaction.
\end{abstract}

DOI: 10.1103/PhysRevB.96.155448

\section{INTRODUCTION}

Few-layer black phosphorus (BP), a two-dimensional semiconductor with a tunable direct band gap, is of growing importance not only for the potential technological applications but also for fundamental condensed-matter studies [1-16]. A layer-controlled small band gap of few-layer BP, being on the order of a few hundreds of $\mathrm{meV}$, permits tuning the chemical potential between the conduction and valence bands by employing the contact metal engineering [2] and the field effect, and thus enables the ambipolar operation of few-layer BP-based devices $[5,17,18]$. In recent years, the development of device fabrication techniques accompanied with the improvement of BP crystal quality has experienced tremendous progress [19-23]. The hole mobility increased from a few hundred to a few thousand $\mathrm{cm}^{2} \mathrm{~V}^{-1} \mathrm{~s}^{-1}$ [24]. And, a further boost of the hole mobility up to $45,000 \mathrm{~cm}^{2} \mathrm{~V}^{-1} \mathrm{~s}^{-1}$ has been made with BP flakes encapsulated between hexagonal boron nitride (h-BN) in vacuum [25]. This high carrier mobility oscillations has enabled the observation of Shubnikov-de Haas oscillations and the quantum Hall effect in hole-doped ( $p$-type) BP. In spite of these achievements, the performance of electron-doped (n-type) BP-based devices remains poor and therefore the electron quantum transport is less explored. According to the theoretical models, the electrons in BP ought to exhibit distinguishable many-body phenomena due to the expected large electron mass [4]. Hence, there is a quest to improve the quality of electron-doped BP crystal and the performance of the devices made thereof, which will eventually lead to the emergence of new quantum phenomena in $n$-type BP.

\footnotetext{
*Corresponding author: phwang@ust.hk
}

In this paper we investigate quantum transport in BP flakes encapsulated in $\mathrm{h}-\mathrm{BN}$ in vacuum conditions. Using contact metal engineering $[22,26]$ and the field effect, we control the charge-carrier type from $p$ - to $n$ type. These ambipolar devices demonstrate high-quality quantum transport characteristics both for electrons and holes. Although quantum transport has been reported in a similar BP two-dimensional hole gas (2DHG) [25], by applying a magnetic field up to $30 \mathrm{~T}$ the zero-resistance states of $R_{x x}$ accompanied with quantized $R_{x y}$ observed in this work demonstrates a fully developed quantum Hall effect in the BP 2DHG. In the same devices, the electrons exhibit clearly quantized $R_{x y}$ accompanied with $R_{x x}$ oscillations, which signal the onset of the quantum Hall effect in $n$-type BP, including spin-resolved states. On the basis of these observations, we extract the electron effective mass, spin susceptibility, and Landé $g$ factor.

The core part of the paper (Sec. II) is organized as follows: in Sec. II A we present the transport characteristics of two BP devices in zero magnetic field; in Sec. II B we discuss the quantum transport of moderately high-mobility electrons and holes in device 1; in Secs. II C and II D we present data on the record-high-mobility device 2 , describing the measurement of the electron spin susceptibility via the coincidence method (Sec. II C), and the study of the integer quantum Hall effect in the $p$-doped regime (Sec. II D).

\section{EXPERIMENTAL RESULTS AND DISCUSSION}

\section{A. Transport characteristics of the ambipolar BP devices}

Figure 1(a) displays the schematic side- and top views of the Hall-bar devices fabricated from a h-BN/BP/h-BN heterostructure which is assembled in vacuum to reduce the surface absorption and to minimize the amount of impurities on 
(a)

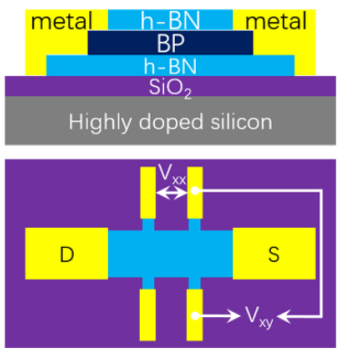

(c)

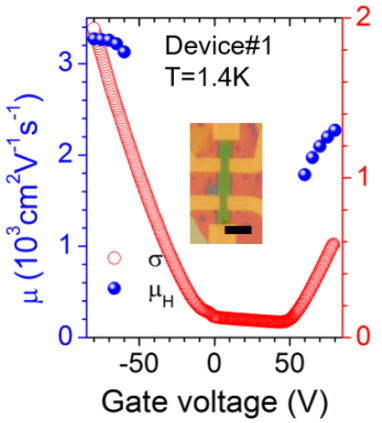

(b)

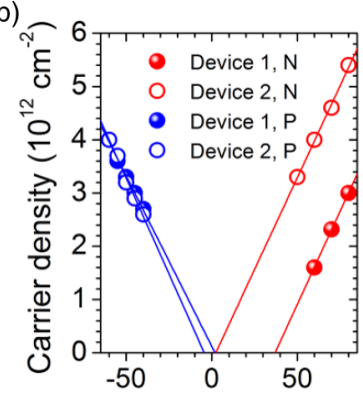

(d)

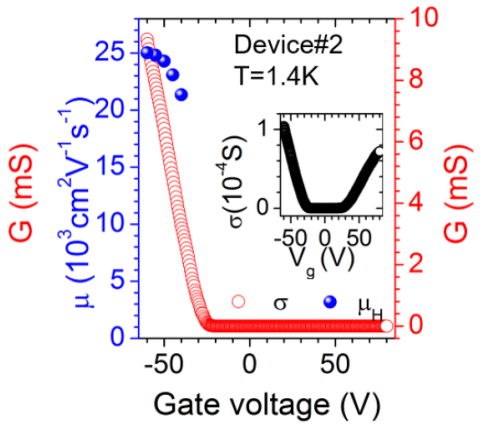

FIG. 1. Transport characteristics of ambipolar BP field-effect devices. (a) Schematic view of BP field-effect transistors and measurement configuration. (b) The hole (blue) and electron (red) densities obtained from the oscillation periods at varying gate voltages. (c), (d) Conductance-gate-voltage characteristics (red) of device 1 (c) and device 2 (d) at $1.4 \mathrm{~K}$ measured in a four-terminal configuration. The blue solid dots represent the Hall mobility. The inset of (c) shows a photo of device 1 taken under microscope. The scale bar is $5 \mu \mathrm{m}$. The inset of (d) shows the two-terminal conductance of device 2 .

the BP interfaces [25]. The Ohmic contacts are made of either $\mathrm{Ti} / \mathrm{Au}$ (device 1 ) or $\mathrm{Cr} / \mathrm{Au}$ (device 2 ) because of their work functions being close to conduction- and valence-band edges of $\mathrm{BP}$, respectively $[22,26,27]$. All the conductance channels are along the $X$ direction (Supplemental Material [28]). The electrical transport measurements are performed with standard lock-in techniques (excitation frequency: $4.579 \mathrm{~Hz}$ ) [22]. For clarity, properties of two devices are summarized in Table I.

Figure 1(b) shows the dependence of the charge-carrier density for both devices on the gate voltage applied between the conducting substrate acting as a gate electrode and one of the Ohmic contacts. The hole (electron) density $p(n)$ is estimated for selected gate-voltage values either from the Hall effect or from the period of magnetoresistance oscillations. This demonstrates a good tuning capability between the electron and hole charge-carrier types. Figure 1(c) depicts the fourterminal conductance $G$ of device 1 as a function of the

gate voltage $V_{g}$ at temperature $T=1.4 \mathrm{~K}$. The conductance increase for positive and negative gate voltages is accompanied with an increasing Hall mobility $\mu_{H}=\frac{G}{p(\text { or } n) \cdot e} \frac{L}{W}$. Here $L$ and $W$ are the length and the width of the device, respectively. The Hall mobility for holes and electrons reaches 3150 and $2150 \mathrm{~cm}^{2} \mathrm{~V}^{-1} \mathrm{~s}^{-1}$ at $T=1.4 \mathrm{~K}$, respectively. Figure 1(d) summarizes the field-effect characteristics of device 2. The transport characteristics measured in a four-terminal configuration show a typical $p$-type unipolar conductance. The channel conductance increases with the decreasing gate voltage and the Hall mobility reaches $25600 \mathrm{~cm}^{2} \mathrm{~V}^{-1} \mathrm{~s}^{-1}$ at $T=1.4 \mathrm{~K}$ - comparable with a previous report [25]. For a positive gate voltage, the four-probe measurement of $V_{x x}$ fails, likely due to malfunctioning of one of the Ohmic contacts used as voltage probes. Note that the energy difference between the work function of $\mathrm{Cr}$ and the Fermi level of $\mathrm{BP}$ is rather large for the $n$-type channel. Nonetheless, the device can be characterized in a two-point measurement. The inset of Fig. 1(d) depicts the two-point conductance, which yields a lower limit for the field-effect mobility of 850 and $420 \mathrm{~cm}^{2} \mathrm{~V}^{-1} \mathrm{~s}^{-1}$ for holes and electrons, respectively. These values are obviously underestimated due to the contact resistance. In device 2 the Hall mobility for holes obtained in four- and two-point measurements differs by a factor of 30 . Thus, assuming the same factor underestimating the electron mobility in two-point measurements, we can infer the electron Hall mobility to be on the order of $12600 \mathrm{~cm}^{2} \mathrm{~V}^{-1} \mathrm{~s}^{-1}$.

The different Hall mobilities in devices 1 and 2 are likely to result from an enhanced scattering rate due to the impurities and imperfections of the $\mathrm{SiO}_{2}$ substrate. Indeed, the bottom h-BN layer of device 1 is only $4.6 \mathrm{~nm}$ thick, and is thinner than the 12.3-nm-thick h-BN layer in device 2. Thus, the charge-carrier transport in device 1 is more susceptible to the scattering centers of the $\mathrm{SiO}_{2}$ substrate than device 2. In the following sections we demonstrate the ambipolar quantum transport characteristics of both devices in a magnetic field.

\section{B. Ambipolar magnetotransport in BP}

Figure 2(a) displays the dependence of $R_{x x}$ and $R_{x y}$ on the magnetic field for hole-type carriers obtained upon the application of a back-gate voltage of $-60 \mathrm{~V}$ on device 1 . The magnetotransport for electron-type carriers in the same device is presented in Fig. 2(b) when a back-gate voltage of $+80 \mathrm{~V}$ is applied. The onset of $R_{x x}$ oscillations at $5 \mathrm{~T}$ for holes and $10 \mathrm{~T}$ for electrons corresponds to a quantum mobility of 2000 and $1000 \mathrm{~cm}^{2} \mathrm{~V}^{-1} \mathrm{~s}^{-1}$, respectively. These values approximate the Hall mobility [Fig. 1(c)] and indicate that the large-angle scattering events dominate, likely due to thin bottom h-BN flakes, over the small-angle scattering processes in this device [25,29-32]. The insets in Figs. 2(a) and 2(b) show the fast

TABLE I. Summary of device properties.

\begin{tabular}{lcccc}
\hline \hline Device no. & $\begin{array}{c}\text { Contact } \\
\text { metals }\end{array}$ & $\begin{array}{c}\text { Thickness of } \\
\text { BN layer }(\mathrm{nm})\end{array}$ & $\begin{array}{c}\text { Mobility from four-point } \\
\text { characterization }\left(\mathrm{cm}^{2} / \mathrm{Vs}\right)\end{array}$ & $\begin{array}{c}\text { Mobility from two-point } \\
\text { characterization }\left(\mathrm{cm}^{2} / \mathrm{Vs}\right)\end{array}$ \\
\hline 1 & $\mathrm{Ti} / \mathrm{Au}$ & 4.6 & $\begin{array}{c}p \text { type: } 3150 / n \text { type: } 2150 \\
p \text { type: } 25600 / n \text { type: } 12600^{\mathrm{a}}\end{array}$ & $p: 850 / n: 420$ \\
2 & $\mathrm{Cr} / \mathrm{Au}$ & 12.3 & NA & $p$ \\
\hline \hline
\end{tabular}

${ }^{a}$ This value is not directly measured from transport properties but estimated by considering the underestimation effect of contact resistance. 

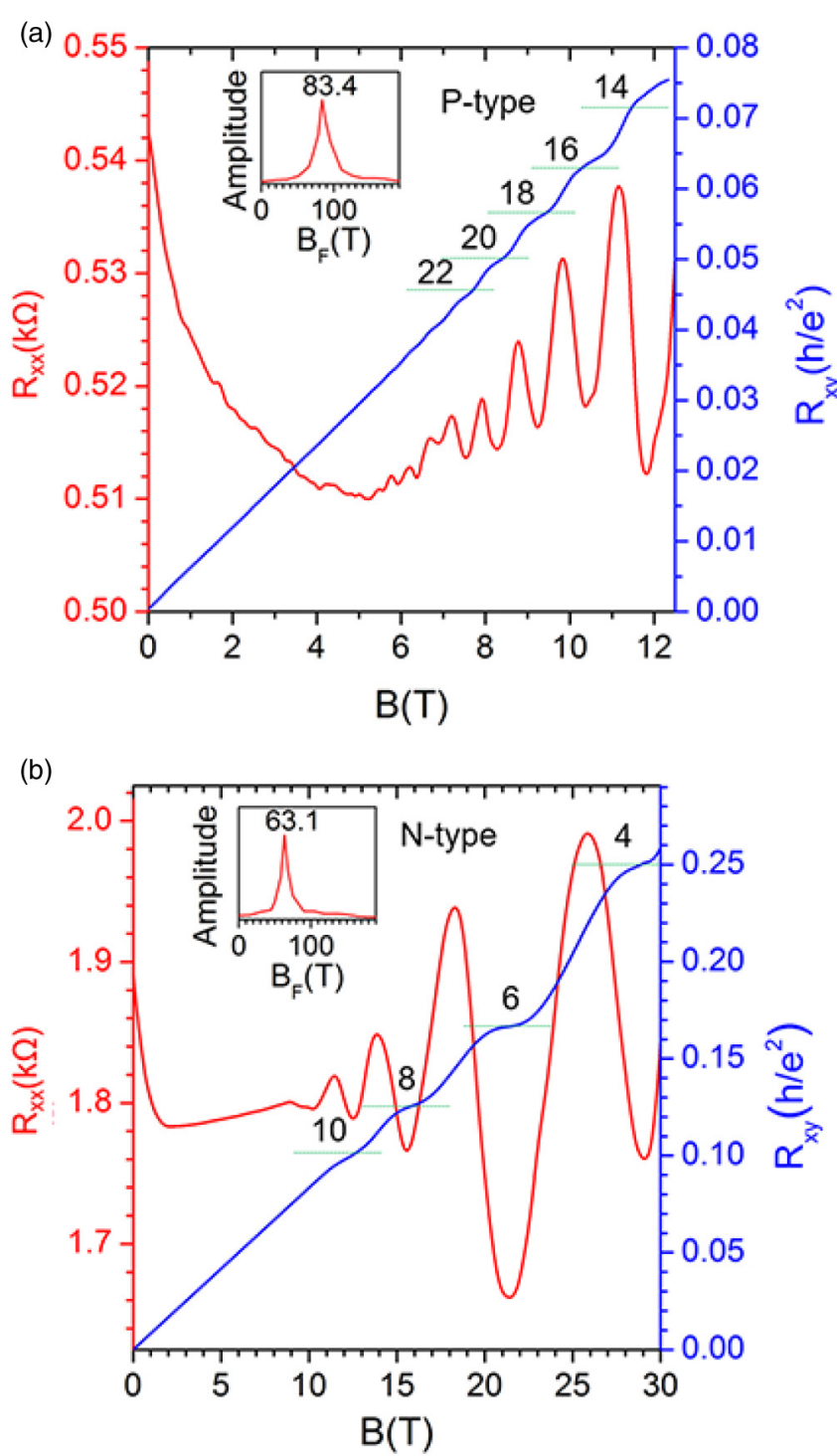

FIG. 2. Quantum Hall effect in the ambipolar BP, device 1. (a), (b) Hall resistance $R_{x y}$ and magnetoresistance $R_{x x}$ as a function of magnetic field $B$ at temperature $T=1.4 \mathrm{~K}$ and gate voltage of $V_{g}=$ -60 and $+80 \mathrm{~V}$, respectively. The integer number and short dashed green lines mark the filling factors $v$.

Fourier transformation (FFT) of $R_{x x}$ as a function of $1 / B$. The carrier concentrations extracted from the FFT frequencies agree well with those determined from the Hall resistance manifesting the absence of another parallel conducting channel in both $n$ - and $p$-type transport. At high magnetic fields, $R_{x y}$ exhibits plateau formations corresponding to the quantum Hall resistance $h / v e^{2}$, where $v$ is an integer number. These plateaus align well with $R_{x x}$ minima. Thus the magnetotransport signals the (onset of the) quantum Hall effect for $p$-type ( $n$-type) charge carriers in the BP device.

Figure 3(a) shows the temperature dependence of the $R_{x x}$ oscillatory component for the $n$-type conduction in device 1 and reveals the damping of the oscillation amplitude with temperature. This damping behavior follows the Lifshitz-Kosevitch formalism $\Delta R \sim \frac{\lambda(T)}{\sinh [\lambda(T)]}$, where $\lambda(T)=$ $2 \pi^{2} k_{B} T m^{*} / \hbar e B$ is the temperature damping factor, and allows the estimation of the electron effective mass. Figure 3(b) shows a fairly good fitting of oscillation amplitude damping using the Lifshitz-Kosevitch formalism. The extracted electron effective mass is $0.39 \pm 0.03 m_{0}$ and is independent of the magnetic field as shown in Fig. 3(c). This value is consistent with the previous report [22] and the theoretical prediction [4].

The magnetotransport for electron- and hole-type charge carriers in device 1 shows the conductance quantization at only even filling factors, which suggests that the spin degeneracy is not lifted even if the applied magnetic field is very large. The discussion of the transport characteristics of device 2 presented below may suggest that the high disorder of device 1-reflected in a rather low Hall mobility and the onset of $R_{x x}$ oscillations at a rather high magnetic field-can likely account for not observing the lifting of the spin degeneracy. Accordingly, device 1 does not allow one to access the spin properties of electrons in BP, which are instead revealed in the study of device 2 .

\section{Spin susceptibility of electrons}

In the following we turn our attention to device 2 , fabricated with chromium Ohmic contacts, which has a much higher charge-carrier mobility than device 1 . Considering the rich research reports in the literature about $p$-type BP, we start by discussing in this section the quantum transport characteristics in the electron-doped regime. The bottom trace of Fig. 4(b) exemplifies the oscillation component of the two-terminal magnetoresistance $\Delta R$ with $V_{g}=+80 \mathrm{~V}$ and $T=1.4 \mathrm{~K}$, measured with the magnetic field perpendicular to the plane of two dimension electron gas (2DEG) (i.e., at zero tilt angle) in device 2. The charge-carrier density [see Fig. 1(b)] is determined from the period of the quantum oscillations. The minima of oscillations correspond to the integer filling factors and the splitting of quantum oscillations starts at $12 \mathrm{~T}$. This splitting signals the lifting of spin degeneracy, which enables exploring the spin properties of electrons in BP. We now utilize this fact to measure the electron spin susceptibility by bringing the spin-resolved Landau level to coincidence-the so-called coincidence technique [25,33-39] This method profits from the fact that the Zeeman energy $E_{z}=g \mu_{B} B_{T}$ is given by the total magnetic field $B_{T}$, while the cyclotron energy $E_{c}=$ $\hbar e B_{\perp} / m^{*}$ scales with the magnetic-field component normal to the sample plane. Thus $E_{c}$ and $E_{z}$ can be controlled individually by tilting the sample in the magnetic field. At certain angles $\theta_{i}$, the Zeeman energy is an integer of cyclotron energy, i.e., $E_{z}=i E_{c}$, and the spin-resolved Landau levels overlap as shown in Fig. 4(a). The spin susceptibility $\chi_{s}$ can be extracted from the coincidence condition $\chi_{s}=m^{*} g^{*}=$ $2 i \cos \left(\theta_{i}\right)$. Figure 4(b) displays the evolution of the oscillatory part of the two-point resistance under a constant $B_{\perp}$ as $B_{T}$ and/or the tilt angle increases. A coincidence event in the experiment is marked by the vanishing of a quantum oscillation component at even/odd filling factors, i.e., the disappearance of the splitting features. As shown in Fig. 4(b), when the tilt angle approaches $\theta_{1}=56.4^{\circ}$, even- $v \Delta R$ deep features gradually become weak and finally disappear at $\theta_{1}$. When the tilt angle increases beyond $\theta_{1}$ the deep feature reappears again. The same evolution takes place at $\theta_{2}=73.9^{\circ}$. The oscillation amplitude reaches its maxima at both $\theta_{1}$ and $\theta_{2}$, further confirming the 
(a)

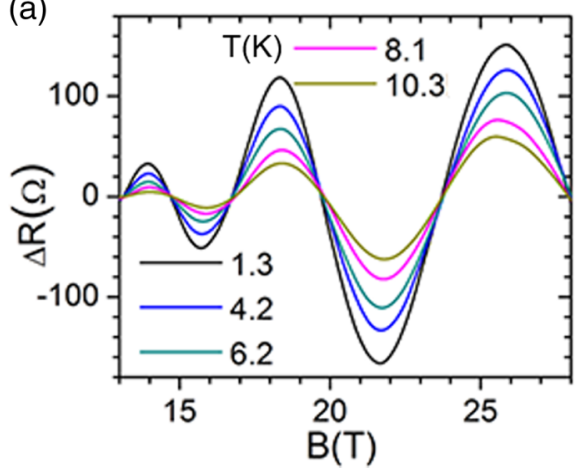

(b)

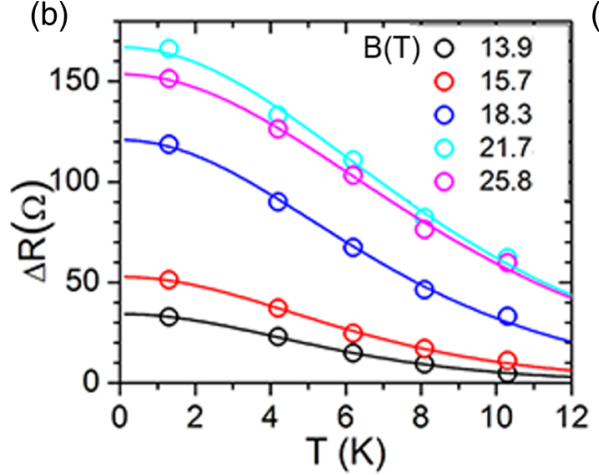

(c)

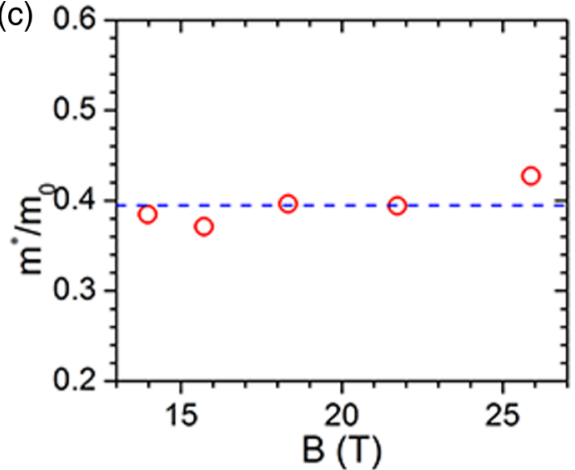

FIG. 3. Cyclotron mass of electrons in BP, device 1. (a) The oscillation components of $n$-type BP are damped with the increasing temperature. (b) The temperature damping of oscillation components is fitted by the Lifshitz-Kosevitch formula. The solid lines represent the fitting results. (c) Effective mass of $n$-type BP obtained from the fitting results shown in (b).

(a)

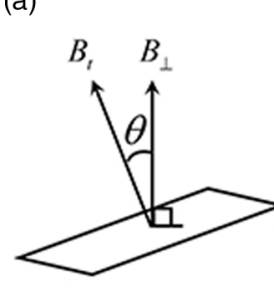

(b)

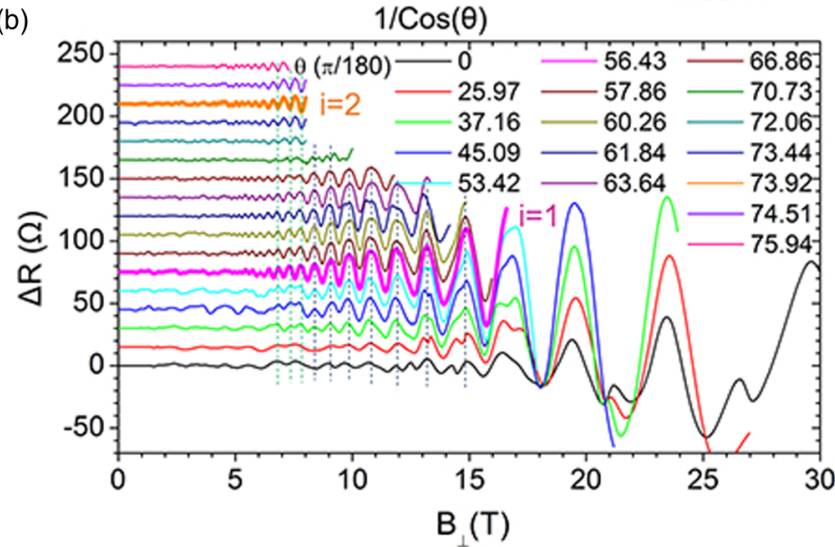

FIG. 4. Landau level crossing of electrons under tilted magnetic field, device 2. (a) Left panel: The tilt angle $\theta$ is defined between the total magnetic field $B_{T}$ and the magnetic-field component $B_{\perp}$ normal to the charge carrier's plane. Right panel: Schematic fan diagram showing spin degeneracy lifted Landau levels evolving with the tilt angle. The purple arrow indicates cyclotron energy $E_{c}=\hbar \omega_{c}$ and the dark blue arrow represents the Zeeman energy $E_{z}=g \mu_{B} B$. The states represented by green solid lines are occupied by electrons with spin-up orientation while those represented by blue solid lines are occupied by electrons with spin-down orientation. The vertical solid red line shows the case of $\theta=0$ and the three vertical dashed red lines indicate the first, second, and third coincidence angles, respectively. (b) Magnetoresistance for different tilt angles when a gate voltage $V_{g}=+80 \mathrm{~V}$ is applied at the temperature $T=1.4 \mathrm{~K}$. The magnetotransport traces recorded at the coincidence events $i=$ 1 and $i=2$ are emphasized with bold lines. (c) $1 / \cos (\theta)$ values for the identified coincidence angles as a function of $i$. The solid line represents the linear fitting result indicating a spin susceptibility $\chi_{\text {se }}=1.1 \pm 0.03$. coincidence events at these two angles [40]. The coincidence events at $i=1$ and $i=2$ are emphasized by representing $\Delta R$ with bold lines in Fig. 4(b). Figure 4(c) shows $1 / \cos \left(\theta_{i}\right)$ versus $i$. The linear fit of this dependence yields the electron spin susceptibility in black phosphorus, $\chi_{s e}=1.1 \pm 0.03$. This value is $70 \%$ larger than that of $p$-type BP conducting channel $\left(\chi_{s h}=0.64\right)$, illustrating an energetically equidistant arrangement of spin-resolved Landau levels for high filling factors $(v>15)$ at zero tilt angle, which can make $n$-type $\mathrm{BP}$ an interesting platform to explore spin-related phenomena relevant for spintronics. Taking into account the electron effective mass $m^{*}=0.39 m_{0}$, the Landé $g$ factor for $n$-type conducting BP is determined to be $g^{*}=2.8 \pm 0.2$. This value in $n$-type BP is roughly the same one as in $p$-type conducting $\mathrm{BP}(g *=2.5)$ [25]. The enhanced Landé $g$ factor has been observed in 2DEG and $2 \mathrm{DHG}$ of $\mathrm{BP}$ and can originate from the exchange interaction [39-44]. The interaction strength is gauged by the Wigner-Seitz radius (ratio of the Coulomb energy to the kinetic energy) $r_{s}=\frac{m^{*}}{\sqrt{\pi n(\text { orp })} \alpha_{B} \kappa m_{0}}$, where $n$ (or $p$ ) is the carrier density, $\alpha_{B}$ is the Bohr radius, and $\kappa$ is the dielectric constant of the surrounding medium $[45,46]$; the interaction effects are more pronounced in systems with a larger effective mass and lower carrier concentrations. Table II compares the spin susceptibilities for several two-dimensional electron and hole systems with different effective masses but the same $r_{s} \approx 2$, achieved in $n$-type BP at $V_{\mathrm{g}}=+80 \mathrm{~V}$. An enhancement of spin susceptibility by about $50 \%$ compared to the band susceptibility is observed for all systems. This can indicate a similar role played by the electron-electron interaction in different $2 \mathrm{DE} / \mathrm{HG}$ s except $\mathrm{WSe}_{2}$, in which an enhanced susceptibility may arise from the strong spin-orbit coupling. The spin-orbit coupling is also known to modify the $g$-factor value. However, the spin-orbit coupling is small in black phosphorus and therefore should not contribute to $g$-factor enhancement [4]. Moreover, the recent theoretical investigation of $g$ factor in black phosphorus, which is based on multiband k•p calculations, predicts that the Landau level broadening and the gate electric field can affect the $g$-factor value [45].

\section{Quantum Hall effect in $p$-type BP}

Finally, although quantum transport in $p$-type BP has been reported before [25], we believe that it is worthwhile to 
TABLE II. Comparison of spin susceptibility (in units of e/2h) for BP-, WSe $2_{-}^{-}, \mathrm{ZnO}-$, AlAs-, and GaAs-based 2DEGs/2DHGs with carrier density corresponding to $r_{s} \approx 2$ (except $\mathrm{ZnO}$-based system and $p$-type BP system). The spin susceptibility of $\mathrm{ZnO}$-based system is measured at a carrier density corresponding to $r_{s} \approx 8$. For $p$-type BP system, average spin susceptibility for $r_{s}$ ranging from 1.4 to 2 is obtained. The data for monolayer $\mathrm{WSe}_{2}, \mathrm{AlAs}$, and $\mathrm{GaAs}$ are extracted from the formulas and figures of the corresponding references. $\chi_{\text {band }}$ is the band susceptibility and $\chi_{m}$ is the measured susceptibility.

\begin{tabular}{|c|c|c|c|c|c|c|}
\hline 2DE/HGs & Type & $m * / m_{0}$ & $\chi_{\text {band }}$ & $\chi_{m}$ & $\chi_{m} / \chi_{\text {band }}$ & Reference \\
\hline BP & $n$ & 0.39 & 0.78 & $1.1 \pm 0.03$ & $1.41 \pm 0.04$ & This work \\
\hline $\mathrm{BP}$ & $p$ & 0.26 & 0.52 & 0.64 & 1.23 & 25 \\
\hline $\mathrm{WSe}_{2}$ & $p$ & 0.45 & 0.9 & 7.2 & 8.0 & 46 \\
\hline $\mathrm{ZnO}$ & $n$ & 0.3 & 0.6 & 1.77 & 2.95 & 35 \\
\hline AlAs & $n$ & 0.46 & 0.92 & 1.38 & 1.5 & 44 \\
\hline GaAs & $n$ & 0.067 & 0.029 & 0.043 & 1.6 & 42 \\
\hline
\end{tabular}

investigate such phenomenology in a very high-mobility device and make use of strong magnetic fields. The magnetotransport characteristics for holes in device 2 in a magnetic field up to $30 \mathrm{~T}$ are shown in Fig. 5(a). Here the back-gate voltage is $-60 \mathrm{~V}$ and the temperature is $T=1.4 \mathrm{~K}$. At low magnetic fields $(B<3 \mathrm{~T}), R_{x x}$ exhibits nearly zero magnetoresistance, indicating the bare existence of localizations in the sample and demonstrating the unprecedented quality of the BP device. The onset of $R_{x x}$ oscillations starts at $3 \mathrm{~T}$ with the sequence of only even Landau level filling factors. This corresponds to a quantum mobility of $3300 \mathrm{~cm}^{2} \mathrm{~V}^{-1} \mathrm{~s}^{-1}$, which is about eight times lower than the Hall mobility. This suggests that the small-angle scattering by remote charge impurities dominates over the large-angle scattering due to a thick h-BN bottom layer in device 2 . The splitting of $R_{x x}$ oscillations occurs at 10 $\mathrm{T}$ and develops rapidly in the magnetic field, demonstrating the impact of exchange interactions on the splitting, which is associated with lifting the spin degeneracy. The Fourier transform of periodic $R_{x x}$ oscillations on the $1 / B$ axis shows two spectral lines at 85 and $170 \mathrm{~T}$ [inset of Fig. 5(a)] and thus confirms the lifting of spin degeneracy. When the magnetic field reaches $15 \mathrm{~T}$, the zero resistance of $R_{x x}$ as well as the quantized $R_{x y}$ plateaus clearly establish the realization of the quantum Hall effect in $p$-type BP device [47]. By sweeping the gate voltage at $B=30 \mathrm{~T}$ one gains an access to lower filling factors. Figure 5(b) reveals a set of $R_{x y}$ plateaus at values $h / e^{2} v$, where $v$ assumes an integer number from 2 to 5 , accompanied with the zero-resistance $R_{x x}$ states. Thus, the Landau level filling factor $v$ can be unequivocally assigned to each plateau. The quantum Hall-effect observation at both even and odd filling factors confirms the lifting of spin degeneracy at high magnetic field.

Well-developed quantum Hall states enable the quantitative study of the thermal activated transport in our BP device. Figure 6(a) shows the thermal activated transport for filling factors $v=3,4$, and 5 under a perpendicular magnetic field of $30 \mathrm{~T}$. The change of $R_{x x}$ minima with the temperature displayed in Fig. 6(c) follows the Boltzmann law $R_{x x}^{\min } \sim \exp \left(-\Delta E / 2 k_{B} T\right)$, where $\Delta E$ is the effective energy gap of the corresponding state. Since the odd filling factors in black phosphorus are associated with the Zeeman gap, the activation energy of the particular state at odd filling factor is expected to depend linearly on the applied magnetic field. Therefore, the activation energy changes as $\Delta E_{\text {odd }}=g \mu_{B} B-\Gamma$, where $\Gamma$ is the Landau level broadening. (a)

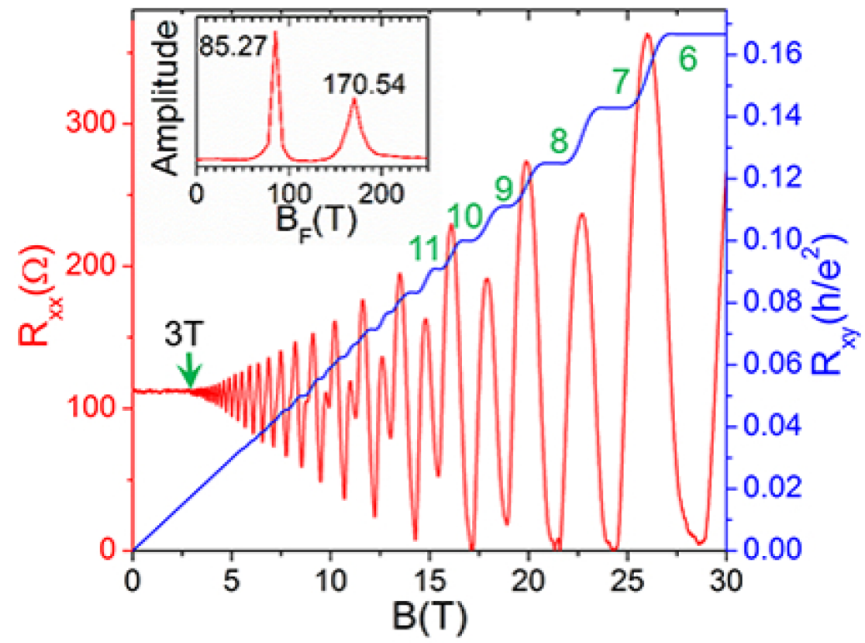

(b)

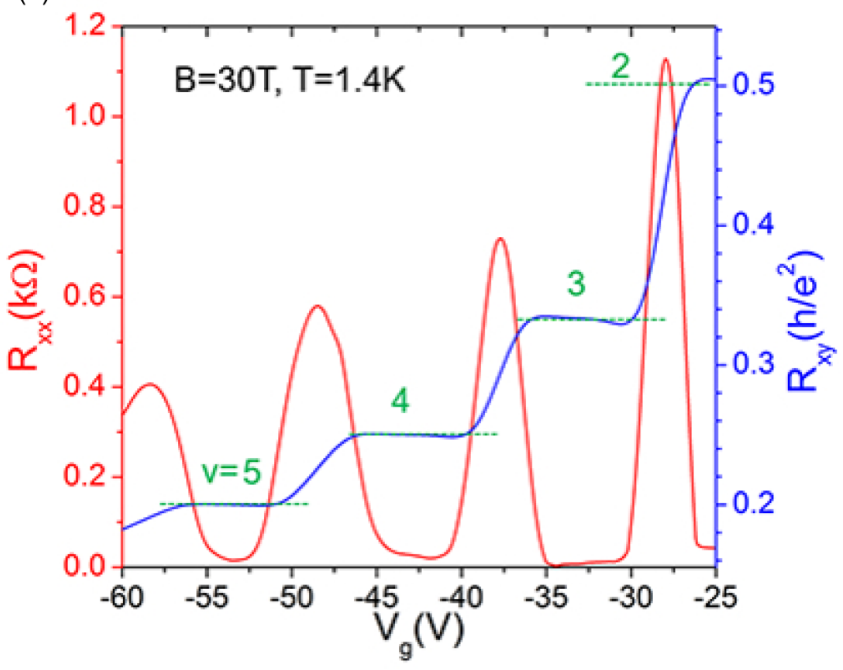

FIG. 5. Quantum Hall effect in $p$-type few-layer BP, device 2. (a) Hall resistance $R_{x y}$ (red) and magnetoresistance $R_{x x}$ (blue) as a function of perpendicular magnetic field $B$ at gate voltage $V_{g}=$ $-60 \mathrm{~V}$ and temperature $T=1.4 \mathrm{~K}$. The onset magnetic field is $3 \mathrm{~T}$. The inset displays the FFT spectrum of $R_{x x}$ versus $1 / B$. (b) $R_{x y}$ and $R_{x x}$ as functions of gate voltages at $T=1.4 \mathrm{~K}$ under a magnetic field of $B=30 \mathrm{~T}$. The integer number in (a) and (b) indicates the filling factors $v$ at quantized $R_{x y}=h / v e^{2}$. 
(a)

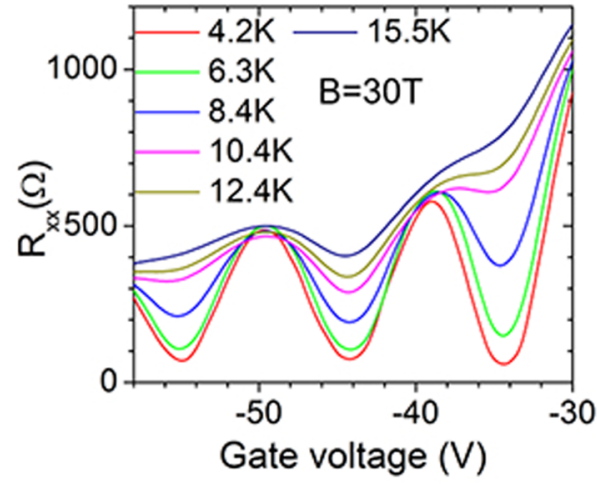

(b)

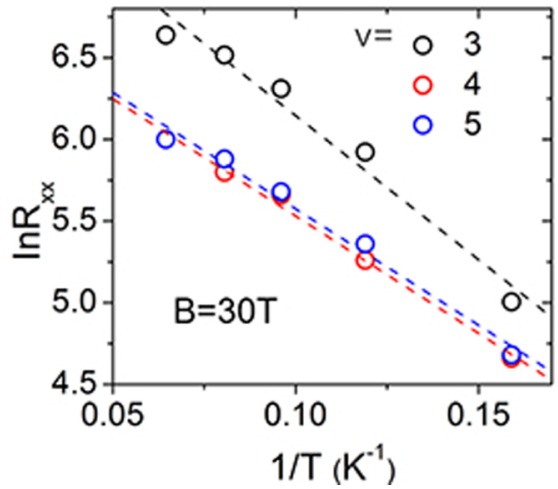

(c)

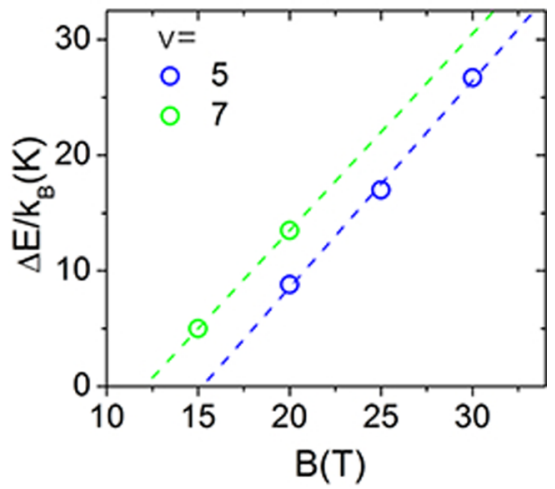

FIG. 6. Landé $g$ factor of holes, device 2. (a) $R_{x x}$ depends on gate voltages under $B=30 \mathrm{~T}$ at varying temperatures. (b) $\ln R_{x x}$ versus $1 / T$ for filling factors $v=3,4$, and 5. The dashed lines are the fitting according to the Boltzmann law $R_{x x}^{\min } \sim \exp \left(-\Delta E / 2 k_{B} T\right)$, where $\Delta E$ is the activation energy. (c) Dependence of energy gaps at filling factors $v=5$ and $v=7$ on the total magnetic field. The dashed lines demonstrate the linear dependence.

Figure 6(c) plots the energy gap $\Delta E$ as a function of magnetic field for filling factors $v=5$ and 7 . The dashed lines are the best linear fits yielding the Landé $g$ factor $2.5 \pm 0.3$ for $v=7$ and $2.7 \pm 0.3$ for $v=5$. These values are in good agreement with the $g$-factor value obtained through the Landau level coincidence technique reported previously [25].

\section{SUMMARY}

Our study demonstrates the emergence of the quantum Hall effect in the ambipolar operation of high-mobility BP field-effect devices, which allow the investigation of both positively and negatively charged carriers. For the hole-doped BP our best field-effect device barely shows localization effects around zero field, thus demonstrating the state-of-the-art quality of BP field-effect transistors. For BP with electron-type carriers, the onset of the quantum Hall effect is observed and the Landau level crossing has been used to estimate the electron Landé $g$ factor $g *=2.8 \pm 0.2$ and the electron mass $m *=(0.39 \pm 0.03) m_{0}$. Thus the electron spin susceptibility $\chi_{s e}=1.1 \pm 0.03$ is larger than the hole spin susceptibility $\chi_{s h}=0.64$ due to a larger electron effective mass and a larger Landé $g$ factor. While the electron and hole masses are consistent with the band structure of black phosphorus, the enhanced Landé $g$ factors of electrons and holes can be the result of exchange interaction, although, as predicted by the theory, also Landau level broadening and the electric field in the BP field-effect device might play an important role. Further studies, especially in different ranges of carrier density, are in order to clarify the origin of the observed phenomenology.

\section{ACKNOWLEDGMENTS}

We acknowledge the support of the HFML-RU/FOM member of the European Magnetic Field Laboratory (EMFL). Financial support from the Research Grants Council of Hong Kong (Projects No. 16302215, No. 16300717, and No. HKU9/CRF/13G), Guangdong-Hong Kong Joint Innovation Project (Grant No. 2016A050503012), and technical support of the Raith-HKUST Nanotechnology Laboratory for the electron-beam lithography facility at MCPF are acknowledged.
[1] F. Xia, H. Wang, and Y. Jia, Nat. Commun. 5, 4458 (2014).

[2] L. Li, Y. Yu, G. J. Ye, Q. Ge, X. Ou, H. Wu, D. Feng, X. H. Chen, and Y. Zhang, Nat. Nanotechnol. 9, 372 (2014).

[3] A. Castellanos-Gomez, L. Vicarelli, E. Prada, J. O. Island, K. Narasimha-Acharya, S. I. Blanter, D. J. Groenendijk, M. Buscema, G. A. Steele, and J. Alvarez, 2D Mater. 1, 025001 (2014).

[4] J. Qiao, X. Kong, Z.-X. Hu, F. Yang, and W. Ji, Nat. Commun. 5, 4475 (2014).

[5] Y. Takao and A. Morita, Physica B+C 105, 93 (1981).

[6] X. Ling, H. Wang, S. Huang, F. Xia, and M. S. Dresselhaus, Proc. Natl. Acad. Sci. U.S.A. 112, 4523 (2015).

[7] H. Yuan, X. Liu, F. Afshinmanesh, W. Li, G. Xu, J. Sun, B. Lian, A. G. Curto, G. Ye, and Y. Hikita, Nat. Nanotechnol. 10, 707 (2015).
[8] M. Buscema, D. J. Groenendijk, G. A. Steele, H. S. van der Zant, and A. Castellanos-Gomez, Nat. Commun. 5, 4651 (2014).

[9] X. Wang, A. M. Jones, K. L. Seyler, V. Tran, Y. Jia, H. Zhao, H. Wang, L. Yang, X. Xu, and F. Xia, Nat Nanotechnol. 10, 517 (2015).

[10] S. Yuan, E. van Veen, M. I. Katsnelson, and R. Roldán, Phys. Rev. B 93, 245433 (2016).

[11] A. S. Rodin, A. Carvalho, and A. H. Castro Neto, Phys. Rev. Lett. 112, 176801 (2014).

[12] M. Buscema, D. J. Groenendijk, S. I. Blanter, G. A. Steele, H. S. van der Zant, and A. Castellanos-Gomez, Nano Lett. 14, 3347 (2014).

[13] H. Liu, Y. Du, Y. Deng, and D. Y. Peide, Chem. Soc. Rev. 44, 2732 (2015).

[14] T. Low, A. S. Rodin, A. Carvalho, Y. Jiang, H. Wang, F. Xia, and A. H. Castro Neto, Phys. Rev. B 90, 075434 (2014). 
[15] T. Low, R. Roldán, H. Wang, F. Xia, P. Avouris, L. M. Moreno, and F. Guinea, Phys. Rev. Lett. 113, 106802 (2014).

[16] H. O. Churchill and P. Jarillo-Herrero, Nat. Nanotechnol. 9, 330 (2014).

[17] Y. Akahama, S. Endo, and S.-i. Narita, J. Phys. Soc. Jpn. 52, 2148 (1983).

[18] V. Tran, R. Soklaski, Y. Liang, and L. Yang, Phys. Rev. B 89, 235319 (2014).

[19] N. Gillgren, D. Wickramaratne, Y. Shi, T. Espiritu, J. Yang, J. $\mathrm{Hu}$, J. Wei, X. Liu, Z. Mao, and K. Watanabe, 2D Mater. 2, 011001 (2014).

[20] X. Chen, Y. Wu, Z. Wu, Y. Han, S. Xu, L. Wang, W. Ye, T. Han, Y. He, and Y. Cai, Nat. Commun. 6, 7315 (2015).

[21] L. Li, G. J. Ye, V. Tran, R. Fei, G. Chen, H. Wang, J. Wang, K. Watanabe, T. Taniguchi, and L. Yang, Nat. Nanotechnol. 10, 608 (2015).

[22] G. Long, S. Xu, J. Shen, J. Hou, Z. Wu, T. Han, J. Lin, W. K. Wong, Y. Cai, and R. Lortz, 2D Mater. 3, 031001 (2016).

[23] V. Tayari, N. Hemsworth, I. Fakih, A. Favron, E. Gaufrès, G. Gervais, R. Martel, and T. Szkopek, Nat. Commun. 6, 7702 (2015).

[24] L. Li, F. Yang, G. J. Ye, Z. Zhang, Z. Zhu, W. Lou, X. Zhou, L. Li, K. Watanabe, T. Taniguchi et al., Nat. Nanotechnol. 11, 593 (2016).

[25] G. Long, D. Maryenko, J. Shen, S. Xu, J. Hou, Z. Wu, W. K. Wong, T. Han, J. Lin, Y. Cai et al., Nano Lett. 16, 7768 (2016).

[26] D. J. Perello, S. H. Chae, S. Song, and Y. H. Lee, Nat. Commun. 6, 7809 (2015).

[27] Y. Cai, G. Zhang, and Y.-W. Zhang, Sci. Rep. 4, 6677 (2014).

[28] See Supplemental Material at http://link.aps.org/supplemental/ 10.1103/PhysRevB.96.155448 for angle-resolved Raman spectrum of BP.

[29] P. T. Coleridge, Phys. Rev. B 44, 3793 (1991).

[30] B. A. Piot, D. K. Maude, M. Henini, Z. R. Wasilewski, K. J. Friedland, R. Hey, K. H. Ploog, A. I. Toropov, R. Airey, and G. Hill, Phys. Rev. B 72, 245325 (2005).

[31] E. H. Hwang and S. Das Sarma, Phys. Rev. B 77, 195412 (2008).
[32] W. Knap, V. Fal'ko, E. Frayssinet, P. Lorenzini, N. Grandjean, D. Maude, G. Karczewski, B. Brandt, J. Łusakowski, and I. Grzegory, J. Phys.: Condens. Matter 16, 3421 (2004).

[33] H. Schumacher, A. Nauen, U. Zeitler, R. Haug, P. Weitz, A. Jansen, and F. Schäffler, Phys. B: Condens. Matter 256, 260 (1998).

[34] Y. Kozuka, A. Tsukazaki, D. Maryenko, J. Falson, C. Bell, M. Kim, Y. Hikita, H. Y. Hwang, and M. Kawasaki, Phys. Rev. B 85, 075302 (2012).

[35] D. Maryenko, J. Falson, Y. Kozuka, A. Tsukazaki, and M. Kawasaki, Phys. Rev. B 90, 245303 (2014).

[36] A. Tsukazaki, A. Ohtomo, M. Kawasaki, S. Akasaka, H. Yuji, K. Tamura, K. Nakahara, T. Tanabe, A. Kamisawa, T. Gokmen, J. Shabani, and M. Shayegan, Phys. Rev. B 78, 233308 (2008).

[37] S. Xu, J. Shen, G. Long, Z. Wu, Z.-q. Bao, C.-C. Liu, X. Xiao, T. Han, J. Lin, Y. Wu et al., Phys. Rev. Lett. 118, 067702 (2017).

[38] F. Fang and P. Stiles, Phys. Rev. 174, 823 (1968).

[39] R. Nicholas, R. Haug, K. v. Klitzing, and G. Weimann, Phys. Rev. B 37, 1294 (1988).

[40] E. V. Kurganova, H. J. van Elferen, A. McCollam, L. A. Ponomarenko, K. S. Novoselov, A. Veligura, B. J. van Wees, J. C. Maan, and U. Zeitler, Phys. Rev. B 84, 121407(R) (2011).

[41] A. A. Shashkin, S. V. Kravchenko, V. T. Dolgopolov, and T. M. Klapwijk, Phys. Rev. Lett. 87, 086801 (2001).

[42] J. Zhu, H. L. Stormer, L. N. Pfeiffer, K. W. Baldwin, and K. W. West, Phys. Rev. Lett. 90, 056805 (2003).

[43] K. Vakili, Y. P. Shkolnikov, E. Tutuc, E. P. De Poortere, and M. Shayegan, Phys. Rev. Lett. 92, 226401 (2004).

[44] T. Gokmen, M. Padmanabhan, E. Tutuc, M. Shayegan, S. De Palo, S. Moroni, and G. Senatore, Phys. Rev. B 76, 233301 (2007).

[45] D. Maryenko, J. Falson, M. S. Bahramy, I. A. Dmitriev, Y. Kozuka, A. Tsukazaki, and M. Kawasaki, Phys. Rev. Lett. 115, 197601 (2015).

[46] H. C. P. Movva, B. Fallahazad, K. Kim, S. Larentis, T. Taniguchi, K. Watanabe, S. K. Banerjee, and E. Tutuc, Phys. Rev. Lett. 118, 247701 (2017).

[47] M. Stone, Quantum Hall Effect (World Scientific, Singapore, 1992). 